\title{
Workload Consolidation using VM Selection and Placement Techniques in Cloud Computing
}

\author{
Monika Patel \\ PG student, \\ Computer Engineering \\ Department \\ S.P College of Engineering \\ Visnagar, Gujarat, India
}

\author{
Hiren Patel, PhD \\ Professor, \\ Computer Engineering \\ Department \\ S.P college of Engineering, \\ Visnagar-384315
}

\author{
Nimisha Patel \\ Research scholar, \\ Rai University, Ahmedabad \\ Associate Professor, CE \\ Department \\ S.P college of Engineering, \\ Visnagar-384315
}

\begin{abstract}
Cloud computing provides a consumer pay-per-use computing model over the Internet using numerous data centers across the globe. Power consumption by the huge data centers in Cloud environment has attracted the attention of research community. Efficient usage of energy in Cloud can be addressed in many facets. Virtual Machine (VM) consolidation is one of the techniques to save or reduce energy in virtualized data centers. VM Migration in Cloud also provides us an opportunity for reducing energy consumption. In this research, we intend to study various VM placements \& selection policies and VM migration algorithms for underloaded and overloaded hosts to reduce energy consumption and SLA violation. We propose a novel method using combination of two methods, Least Increase Power (LIP) consumption with Host Sort and Minimum Correlation Coefficient (MCC) for consolidation of VM placement, placing a migratable VM on a host based on utilization thresholds. The results show performance of each combination of algorithms varies with the changing value of the parameters brings better in terms of energy consumption, VM migration time and SLA violation. The reader may plunge the appropriate method for energy consumption.
\end{abstract}

\section{Keywords}

Cloud Computing; Energy consumption; Virtual Machine consolidation; Virtualization; VM migration; SLA violation; Virtual machine placement

\section{INTRODUCTION}

Cloud computing delivers a pool of abstracted and virtualized resources including computing power, storage, platforms and software applications over the Internet based on users demand [1].This pool of resources is provisioned as a very high availability and Quality of Service (QoS) are offered by the cloud infrastructure provider by means of service level agreements (SLA) [2] These services may be categorized as infrastructure as a Service (IaaS), Platform as a Service (PaaS), Software as a Service (SaaS) [3] and also available in three deployment models viz.

Public, Private and Hybrid. Virtualization is an important feature of cloud computing. In this work, We focus on VM consolidation and migration. Each VM requires a certain amount of resources such as CPU, memory, storage and bandwidth [4]

Virtualization allows one user to create several virtual machines (VMs) on a single physical hardware [5]. One of the important requirements is to provide reliable QOS. It can be defined in terms of SLA that describe such characteristics as maximum throughput, higher response time and minimal latency [6] Virtualization technologies allow improving resource utilization, technically termed VM Consolidation(or Server Consolidation or Workload Consolidation) is an essential part of resource management of virtualized data centers [7]

The main challenges of energy efficiency in Cloud computing is to minimize both resource usage and SLA violations [8]. To address the issue of energy efficiency, we divide servers into 2 states viz: over utilization and under utilization. Under loaded hosts are those hosts which are under utilizing its CPU capacity. All the VMs of such hosts are migrated to those hosts whose capacity is big enough to hold them. So the letter host is switched off to save power. An overloaded host is one which has already crossed its utilization capacity. Migration is done to under loaded hosts [9]

Rest of the paper is organized as follows. Section 2 covers Background theory and Energy model also the SLA violation metrics for VM Consolidation. We discuss related work and summary of the same is present in tabular form in Section 3. Propose work with architecture and Flowchart is present in Section 4. We conclude our work with future direction in Section 5. At the end, all the references used are cited.

\section{BACKGROUND THEORY}

Virtual machine consolidation to make more efficient use of computer resources and prevent servers and storage equipment from being under-utilized and taking too much space. Also it involves reducing the number of server within an organization. It is a technique of maximizing resource utilization while minimizing energy consumption in a virtualized cloud environment [5]

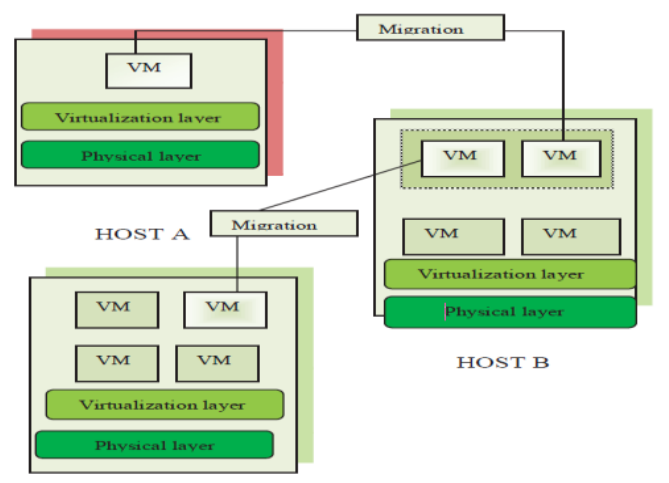

Figure:1 Virtual machine consolidation [10]

Figure1 shows proposed virtual machine consolidation. Each Machine (host) has virtual machine monitor and VM's have guest operating systems. host A has only one VM its CPU utilization value is min but it consumes a lot of power and 
temperature. So the VM of this host is migrated to another host and after that the machine is switched off. By this strategy we can save energy. There are two another hosts in Cloud environment host $\mathrm{B}$ and $\mathrm{C}$. We have to decide which is the best host, on which we can migrate the host A's VM. We found that host $\mathrm{C}$ has four VMs and it is near to its critical temperature but host B has only two VM's and its CPU utilization is also average so this is the best node we will migrate host A's VM on this node and switch off the Host A . As discussed above host $\mathrm{C}$ is overloaded with four VMs, so one $\mathrm{VM}$ of host $\mathrm{C}$ is also migrated on host B.Host $\mathrm{B}$ 's power consumption is less and also temperature of this node is far from critical temperature. So this is the best candidate node for Virtual machine migration.

\subsection{Energy consumption model and SLA violation metrics}

Energy is the capacity to do the work. It is measured in Joule. In energy consumption we use some standard it is called energy consumption model. Also the expected quality level of the service between the consumer and the provider is called Service Level Agreement. SLAs can cover many aspects of the relationship between the Customer and the SP (Service provider). When the SLA is not maintain SLA violation is occur.

Dynamic VM migration and consolidation approaches to reduce both energy consumption and SLA violations [8]. So we formulating a energy consumption model by combining energy usage for computation, for servers switching from active modes, and for communication during VM migrations.

Total energy consumption:

$$
E_{\text {total }}=E_{\text {comp }}+E_{\text {migr }}+E_{\text {swit }}
$$

SLA violation is another important performance metric. The goal of our approach is not only to reduce energy consumption, but also to maintain low SLA violations. We assume SLA is violated when the requested MIPS (Millions of Instructions Per Second) is lower than the allocated MIPS or when the CPU is over-loaded. Two metrics are used for evaluating SLA violations from the perspectives of servers and VMs.

1) $S L A V_{\text {over-loaded }}$ CPU denotes the ratio of SLA violation time and the total active time of servers [8]:

$$
\text { SLAV over-loaded CPU }=\frac{\sum_{i=1}^{N} T v i}{\sum_{i=1}^{N} T a i}
$$

where $N$ is the number of active servers, $T_{\mathrm{vi}}$ is the SLA violation time of the $i$ th server, and $T_{\text {ai }}$ is the total active time of the $i$ th server.

Next, since VM migration causes performance degradation, we also consider SLA violations during VM migrations.

2) 2) SLAVunmet MIPS denotes the ratio of total unmet MIPS and the total requested MIPS during VM migrations:

$$
\text { SLAV }_{\text {unmet MIPS }}=\frac{\sum_{j=1}^{M} \int\left(U r_{j}(t)-U a_{j}(t)\right) d t}{\sum_{j=1}^{M} \int\left(U r_{j}(t) d t\right.}
$$

where $M$ is the total number of migrated $\mathrm{VMs}, U_{\mathrm{rj}}$ is the requested MIPS of the $j$ th VM, and $U_{\text {aj }}$ is the allocated MIPS of the $j$ th VM. The overall SLA violations denoted by $S L A V_{\text {overall }}$ combines the above two SLA violation metrics:

$$
S L A V_{\text {overall }}=\mathrm{SLAV}_{\text {over-loaded } \mathrm{CPU}} \times S L A V_{\text {unmet MIPS }}
$$

Also we summarized total energy consumption is adding the overall energy and it is used with the basic formulation. Then after whole SLA violation measured with multiplying SLAV with over loaded CPU and allocated MIPS.

\section{RELATED WORK}

Cloud providers should reduce their energy consumption of physical machine as much as possible and continue to meet SLAs at the same time. Therefore many researchers have to study energy efficient policies.

Horri, A et .al [12] a novel QOS-aware VMs consolidation approach is proposed that adopts a method based on resource utilization of virtual machine. They have used Local Regression (LR) for overload detection and Minimum Migration Time (MMT) for VM selection but VM-based dynamic threshold (VDT) has been proposed for under load Detection also Utilization and Minimum Correlation (UMC) has been proposed for VM placement. The proposed algorithms significantly reduce number of VM migration, SLAV.

Huang, J et.al [8] proposed Dynamic VM consolidation algorithm which considers both server over-utilization and under-utilization. LR method used for server over- utilization. In over utilization host use VM placement . For VM placement Least Increased Power (LIP) with Host Sort Algorithm , Best Fit Host Algorithm (BFH) and Best Fit VMs Algorithm (BFV) Newly Proposed. In Background theory they all ready discuss proposed new energy consumption model.

Cao, $\mathrm{Z}$ et.al [3] proposed a framework for VM consolidation. They also use many method for overload detection and VM selection. Then they proposed MinPower and MaxUtilization (MPMU) for VM Placement based on MinPower policy. So this result also better compare to MinPower policy.

$\mathrm{Fu}, \mathrm{X}$ et. al [14] proposed a novel VM selection and placement policy which consider degree of resource satisfaction and can reduce energy consumption, VM migration time and SLA violation. First solving the problem is to determine whether a host is overloaded or not. Based on MAD, IR, LR, and LRR solve this problem. Then How to choose a migratable VM when a host's CPU utilization exceeds the upper threshold.. The Meets Performance (MP) methods is proposed for to select in which host to place the VMs. The Power Aware Best Fit Decreasing (PABFD) method is used for VM placement and also Minimum Correlation Coefficient (MCC) is proposed. MCC to search target host that has the least correlation coefficient with the migratable VM. The policies have better performance in the simulated environment.

Beloglazov, A et.al [5] have define the problems consume enormous amounts of electrical energy and carbon dioxide emissions using novel adaptive heuristics for dynamic consolidation of VMs based on an analysis of data from the resource usage by VMs. . The Proposed algorithms reduce energy consumption, while ensuring a high level of the SLA. Use the method for VM consolidation is: 1) In Host Overloading Detection uses MAD, IQR , LR, LRR. 2) In VM selection uses MMT, RC, MC. 3) For determining underloaded hosts a simple approach is used. But those algorithms have failed to get short SLA violation period in each active host and small VMs migrations in virtualized data centers. To solve these problem, they design a novel method for overloaded host and PABFD method for VM placement

Cao, $\mathrm{Z}$ et.al [13] proposed a method for over utilized host and selection in dynamic VM consolidation. They introduce use 
the Median Absolute Deviation (MAD), Interquartile Range (IR), LR, Robust Local Regression (LRR) Minimum Utilization (MU), Random Choice(RC), MMT, Maximum Correlation (MC) for overloaded host and selection of VM. For over utilization host Novel method is proposed also called mean variance(EV) method. In this way author use safe parameter s for determine overloaded host which is different from the other method. Then for selection of VM proposed Extension of Maximum Correlation (MCE) method. It is depend on the Maximum Correlation method.

Then in Table I summarized different techniques that used for virtual machine consolidation.

Table 1Summarized different techniques

\begin{tabular}{|l|l|l|l|c|}
\hline $\begin{array}{l}\text { Related } \\
\text { works }\end{array}$ & $\begin{array}{l}\text { Over } \\
\text { loaded } \\
\text { Host }\end{array}$ & $\begin{array}{l}\text { VM } \\
\text { selection }\end{array}$ & $\begin{array}{l}\text { VM } \\
\text { placement }\end{array}$ & $\begin{array}{l}\text { Under } \\
\text { loaded } \\
\text { Host }\end{array}$ \\
\hline Horri, A[12] & - & - & UMC & VDT \\
\hline Huang, J[8] & LR & MMT & $\begin{array}{l}\text { LIP with } \\
\text { Host Sort } \\
\text { BFH } \\
\text { BFV }\end{array}$ & SM \\
\hline Cao, Z[3] & - & - & MPMU & - \\
\hline Fu, X[14] & - & MP & MCC & - \\
\hline $\begin{array}{l}\text { Beloglazov, } \\
\text { A[5] }\end{array}$ & Novel & $\begin{array}{l}\text { MMT, MC } \\
\text { RC, PABFD }\end{array}$ & $\begin{array}{l}\text { Simple } \\
\text { Method } \\
\text { (SM) }\end{array}$ \\
\hline Cao, Z[13] & EV (Novel) & MCE & - & - \\
\hline
\end{tabular}

\section{OUR CONTRIBUTION}

Various researchers have worked to achieve better performance also one of such significant works has been illustrated by Huang, $\mathrm{J}$ et al [8] and shown in figure 4 .

\subsection{Process}

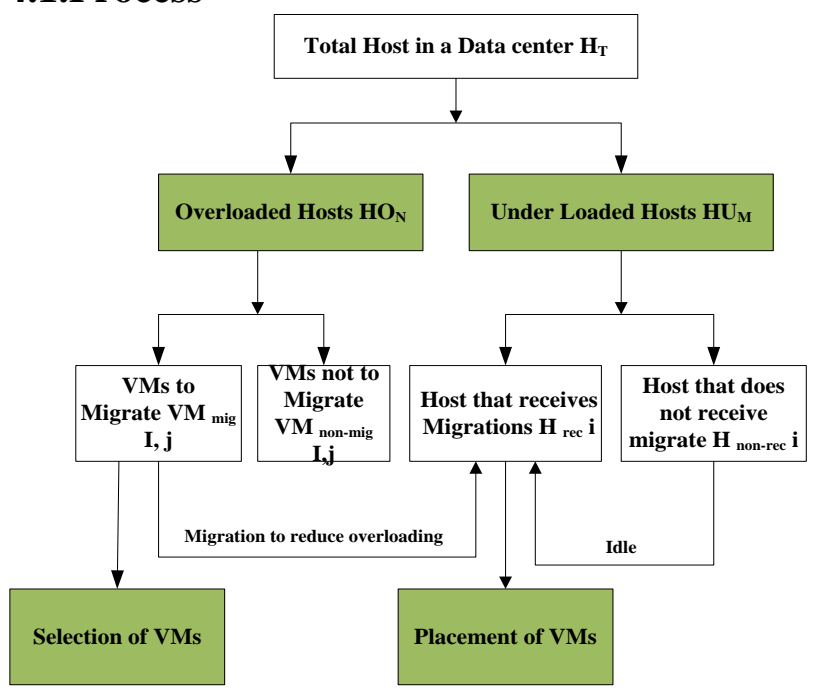

Figure 2VM consolidation Process

In Figure 2 shown we propose a process for VM consolidation. There are many host in data center. These host have under loaded or under loaded find using depending on it's methods.. Each host has many VMs. In over loaded host select the VMs for migrations and also in over loaded host find the place to receive those VMs means placement of VMs. At the end when the under loaded host not receive the VMs then it go to idle state and host is switched off. so save/reduce the energy consumption.

\subsection{Flow Chart}

The proposed system flowchart based on VM consolidation described in [8] is depicted in Fig. 4

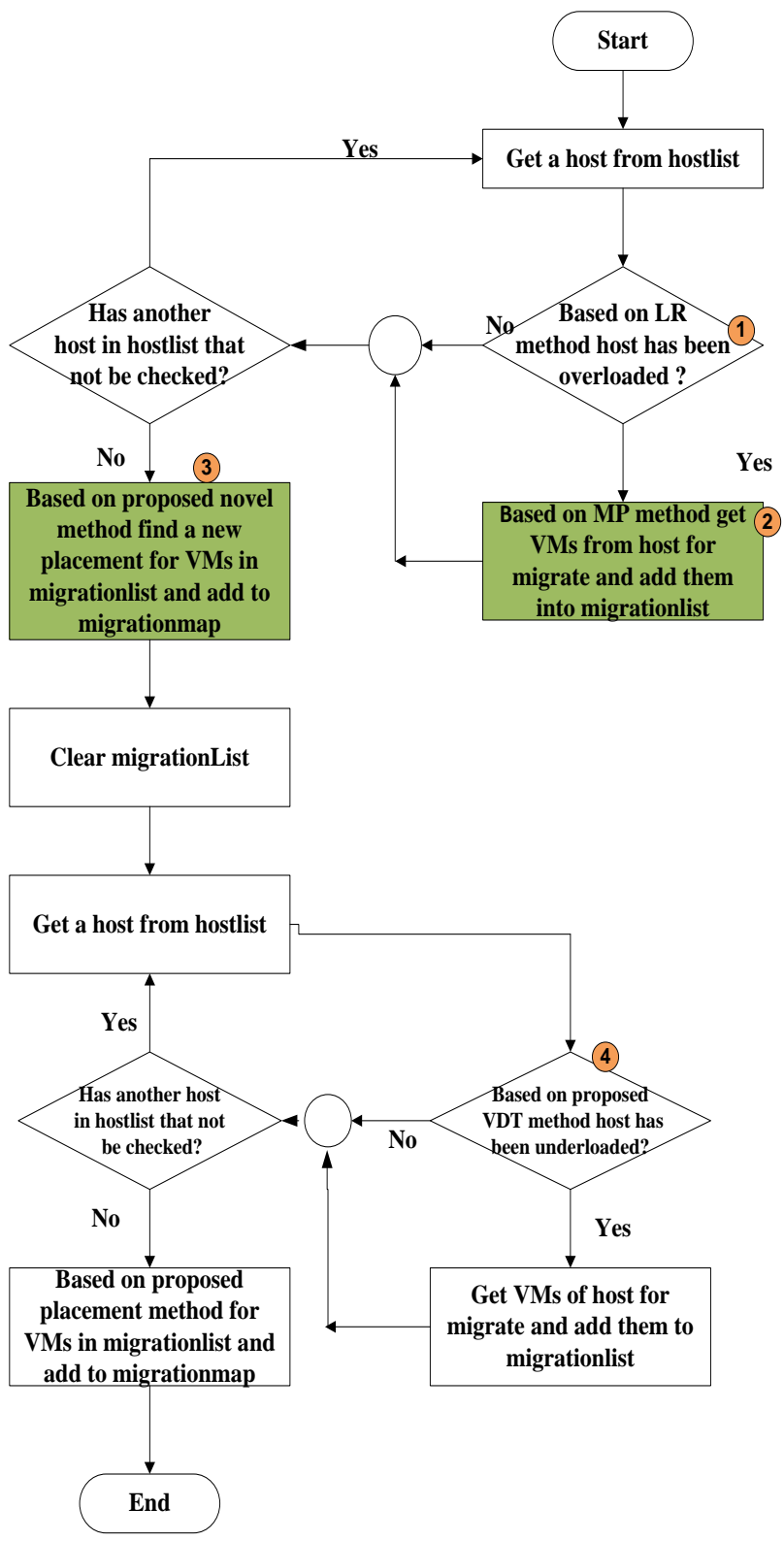

Figure 3Virtual Machine consolidation Flowchart [8]

The number shown in the figure we change the methods for better alternative available options.

At the beginning, find the host from HostList, then after using the Local regression (LR) method determine the host is under loaded or not? When the host is over loaded then, in the next phase using Meets Performance (MP) determine VMs from host for migrate. In this way the policy will make the host's utilization closer to the upper threshold after migration, which will reduce number of migration needed. For Find a new placement for VMs we propose Novel method by the 
combination of two methods, Best Fit Host (BFH) and Best Fit VMs (BFV). BFV and BFH algorithms has achieved better results as it optimally consolidates VMs selected from over utilized hosts to have the highest predicated utilization after migration. At the end of the phase, using VM-Based Dynamic threshold find the under loaded host from the HostList.

\section{CONCLUSION}

It is a challenge for Cloud providers to deal with the trade-off between reducing energy consumption and SLA violation. We have referred quite a good number of research papers and we converse that proper usage of VM selection and placement policies may help reducing number of VM migrations and lowered the energy consumption. We have proposed an idea for VM placement which is combination of two techniques, $\mathrm{BFH}$ and BFV. We have tried to explore new ways using combine two methods which conserve power in Cloud computing. The obtained results optimal performance of each combination of algorithms varies with the changing value of the parameters show that the technique of VM consolidation and switching off the idle host's bring energy saving. The proposed idea is yet to be implemented and tested under simulator or real-time environment before its adaptation or recognition, which is the forthcoming plan of action for us.

\section{REFERENCES}

[1] Foster, I., Zhao, Y., Raicu, I., \& Lu, S. (2008, November). Cloud computing and grid computing 360-degree compared. In Grid Computing Environments Workshop, 2008. GCE'08 (pp. 1-10). Ieee.

[2] Van, H. N., Tran, F. D., \& Menaud, J. M. (2010, July) Performance and power management for cloud infrastructures. In Cloud Computing (CLOUD), 2010 IEEE 3rd International Conference on (pp. 329-336). IEEE.

[3] Cao, Z., \& Dong, S. (2014). An energy-aware heuristic framework for virtual machine consolidation in cloud computing. The Journal of Supercomputing,69(1), 429451 .

[4] Fu, X., \& Zhou, C. (2015). Virtual machine selection and placement for dynamic consolidation in Cloud computing environment. Frontiers of Computer Science, 9(2), 322-330.

[5] Beloglazov, A., \& Buyya, R. (2012). Optimal online deterministic algorithms and adaptive heuristics for energy and performance efficient dynamic consolidation of virtual machines in cloud data centers. Concurrency and Computation: Practice and Experience, 24(13), 1397-1420.
[6] Buyya, R., Yeo, C. S., Venugopal, S., Broberg, J., \& Brandic, I. (2009). Cloud computing and emerging IT platforms: Vision, hype, and reality for delivering computing as the 5th utility. Future Generation computer systems, 25(6), 599-616.

[7] Clark, C., Fraser, K., Hand, S., Hansen, J. G., Jul, E., Limpach, C., ... \& Warfield, A. (2005, May). Live migration of virtual machines. In Proceedings of the 2nd conference on Symposium on Networked Systems Design \& Implementation-Volume 2 (pp. 273-286). USENIX Association.

[8] Huang, J., Wu, K., \& Moh, M. (2014, July). Dynamic Virtual Machine migration algorithms using enhanced energy consumption model for green cloud data centers. In High Performance Computing \&Simulation(HPCS),2014 International Conference on (pp. 902-910). IEEE.

[9] Arzuaga, E., \& Kaeli, D. R. (2010, January). Quantifying load imbalance on virtualized enterprise servers. In Proceedings of the first joint WOSP/SIPEW international conference on Performance engineering (pp.235-242). ACM.

[10] Kinger, S., \& Goyal, K. (2013). Energy-efficient CPU utilization based virtual machine scheduling in Green clouds.

[11] Beloglazov, A., \& Buyya, R. (2010, November). Adaptive threshold-based approach for energy-efficient consolidation of virtual machines in cloud data centers. In Proceedings of the 8th International Workshop on Middleware for Grids, Clouds and e-Science (Vol. 4). ACM.

[12] Horri, A., Mozafari, M. S., \& Dastghaibyfard, G. (2014). Novel resource allocation algorithms to performance and energy efficiency in cloud computing. The Journal of Supercomputing, 69(3), 1445-1461

[13] Cao, Z., \& Dong, S. (2012, December). Dynamic VM consolidation for energy-aware and SLA violation reduction in cloud Computing. In Parallel and Distributed Computing, Applications and Technologies (PDCAT), 2012 13th International Conference on (pp. 363-369). IEEE

[14] Fu, X., \& Zhou, C. (2015). Virtual machine selection and placement for dynamic consolidation in Cloud computing environment. Frontiers of Computer Science, 9(2), 322-33 